\title{
Early Events of Enterotoxigenic Escherichia coli Colonization on Gut Barrier Function: No Longer UndETECted?
}

\author{
Juan María Díaz Carrasco ${ }^{1,2}$ - Mariano Enrique Fernández-Miyakawa ${ }^{1,2}$
}

Published online: 6 February 2017

(c) Springer Science+Business Media New York 2017

Post-weaning diarrhea (PWD), an important enteric disease of pigs that usually occurs shortly after weaning, is characterized by watery diarrhea, dehydration, loss of body weight, and the death of affected animals. It is considered a multifactorial disease associated with proliferation of enterotoxigenic Escherichia coli (ETEC) in the intestinal tract of infected pigs. In animal production, oral antibiotics are commonly used to control PWD. Since antimicrobial resistance in pathogenic bacteria is a global threat, increasing attention is being paid to the prudent use of antibiotics in food-producing animals. Therefore, there is an urgent need for novel and rational strategies helping to prevent PWD and the concomitant reduction of antibiotic use.

In humans, diarrhea continues to be a leading cause of death in children $<5$ years of age with ETEC the most common bacterial cause of children's diarrhea. ETEC infection is also the most important cause of travelers' diarrhea. The virulence of ETEC, transmitted by food or water contaminated with animal or human feces, is believed to be associated with the production of fimbrial adhesins and enterotoxins, including heat-stable enterotoxins $\left(\mathrm{ST}_{\mathrm{a}}\right.$ and $\left.\mathrm{ST}_{\mathrm{b}}\right)$ and heat-labile enterotoxin (LT), which disrupt intestinal fluid homeostasis and promote hypersecretion of fluid and electrolytes through activation of

Mariano Enrique Fernández-Miyakawa

fernandezmiyakawa.m@inta.gob.ar

1 Instituto de Patobiología, Centro Nacional de Investigaciones Agropecuarias, Instituto Nacional de Tecnología

Agropecuaria, Calle Las Cabañas y Los Reseros s/n, Casilla de Correo 25, 1712 Castelar, Buenos Aires, Argentina

2 Consejo Nacional de Investigaciones Científicas y Técnicas, Godoy Cruz 2290, 1425 Ciudad Autónoma de Buenos Aires, Argentina adenylate cyclase (by LT) or guanylate cyclase (by $\mathrm{ST}_{\mathrm{a}}$ ) in small intestine mucosal cells [1].

In this issue of Digestive Diseases and Sciences, Lodemann and colleagues [2] report the interesting observation that ETEC infection elicits early effects on barrier and transport function of piglet jejunal tissues that are modulated by feeding piglets and their mother sows with probiotic strains. These findings, which provide evidence regarding the early steps of ETEC pathogenesis, support a potential mechanism for the favorable action of probiotic strains in the small intestine.

Using electrophysiological studies of Using-chambered swine jejunum, a rapid increase in jejunal electrical resistance was observed in vitro after the intestinal mucosa was exposed to a relatively high concentration of ETEC, accompanied by a decrease in the rate of mucosal to serosa passage of fluorescein, a small molecule used as permeability marker, consistent with decreased transmucosal permeability to ions and to small organic molecules. This observation suggests that the small intestine colonized by E. coli rapidly responds with a tightening of the epithelial barrier, potentially useful in the prevention of consequent translocation of bacteria, bacterial endotoxins, or environmental antigens across the gut wall as well as reducing the outflow of metabolites needed for luminal bacteria growth. Although the most commonly known response of the intestine to a wide range of stressors is the increase of intestinal permeability and conductance, the small intestinal mucosa resistance enhancement has been also observed with other E. coli and Salmonella strains in other animal species [3]. It is probable that small intestine responds to bacterial lipopolysaccharide (LPS), since Yorkshire-bred pigs responded to LPS by increasing mucosal small intestinal resistance [4]. Nonetheless, this change in epithelial resistance could depend on many other genetic or 
physiological factors since resistance of the small intestine of Meishan-bred pigs did not change with LPS. This potential defensive response could be selected during evolution to elude or restrict colonization of small intestine by Gram-negative bacteria, probably through the recognition of distinctive molecules such as LPS or adhesins.

Homeostasis of fluid in the gastrointestinal tract essentially depends on active ion transport. Measurement of electrical parameters under short-circuit current $I_{\mathrm{sc}}$ in an Ussing chamber is a sensitive and powerful tool to study physiological changes in epithelial tissues. Under the experimental conditions used in the present report, changes in $I_{\mathrm{sc}}$ fundamentally represent the active transport of ions across the epithelium. Although no significant changes of $I_{\mathrm{sc}}$ were observed between control and ETEC-infected tissues, the author states that $I_{\mathrm{sc}}$ appeared to increase in parts of the jejunal epithelia in the presence of ETEC. The physiological significance could be attributed to subsegmental differences in intestinal transport physiology. In previous studies, using a different approach as a perfusion technique, ETEC infection of piglet jejunal segments significantly reduced net absorption of fluid, $\mathrm{Na}^{+}$, and $\mathrm{Cl}^{-}$ and increased net secretion of $\mathrm{K}^{+}$compared with controls [5]. In the present report by Lodemann et al., the responses of $I_{\mathrm{sc}}$ after stimulation with the secretagogue $\mathrm{PGE}_{2}$ or glucose were reduced in ETEC-treated jejunum, reinforcing the proposition that transport functions were impaired. The potential mechanism could be attributed to $\mathrm{Na}^{+}$-coupled glucose absorption, although alternative explanations such as an inhibition of $\mathrm{NaCl}$ absorption at the villus tip or increased $\mathrm{Cl}^{-}$secretion could be supported by the available information. Multiple combinations of $\mathrm{LT}, \mathrm{ST}_{\mathrm{a}}$, and $\mathrm{ST}_{\mathrm{b}}$ are produced by ETEC strains associated with diarrheal disease in pigs. Indeed, the most common ETEC strain isolated from diarrheic pigs produces $\mathrm{LT}$ and $\mathrm{ST}_{\mathrm{b}}$, both associated with ETEC-related diarrhea [6]. Of the effects of bacterial enterotoxins on gut ion secretion, the pig jejunum is particularly sensitive to the secretory action of the heatstable enterotoxins [7].

Tight junctions (TJ) are dynamic, multiprotein intercellular adhesive contacts between epithelial cells. Integral membrane proteins that comprise the TJ include claudins; differential expression of the claudin family members is associated with epithelial properties including barrier tightness, strand architecture, and paracellular ion permeability. After the initial tightening of the epithelial barrier by ETEC infection, mucosal resistance decreased to initial levels although the expression of the tight junctional proteins claudin-1, 2, 3, and 7 was unaffected. Nevertheless, expression of claudin- 4 was reduced in control piglet tissues, suggesting that ETEC alters the structure and integrity of epithelial tight junctions. Although the function of claudin-4 is incompletely understood and varies with cell and tissue localization, claudin- 4 protein is considered a TJ-sealing claudin with augmented expression correlated to enhanced epithelial barrier properties and restricted $\mathrm{Na}^{+}$ flux [8].

The intestinal barrier, mainly formed by epithelial cells, maintains homeostasis in the intestine in a continuous cooperative process with the innate immune system [9]. Mucosal inflammation is associated with decreased claudin-4 expression. Also, proinflammatory cytokine exposure during mucosal inflammation disrupts physiological epithelial TJ structure and composition, increasing paracellular permeability [8]. In previous in vivo studies, ETEC infection increased the expression of the proinflammatory cytokines interferon (IFN)- $\gamma$, tumor necrosis factor (TNF)$\alpha$, and interleukin (IL)- 6 in the ileum of pigs [10] or IL-8 in the jejunum of piglets [11]. Also, in the present report, in vitro jejunal ETEC infection induced a proinflammatory response of the epithelia, as the mRNA expression of IL-8, TNF- $\alpha$, and the inflammasome-associated gene NLRP3 was enhanced in those piglet tissues, suggesting that changes in epithelial permeability are associated with proinflammatory signals triggered by $E$. coli.

The probiotic strain Enterococcus faecium used by Lodemann et al. [12] in their studies improves the outcome of acute diarrhea in pigs. Although the in vivo pretreatment of pigs with E. faecium did not modify the observed tightening of the epithelial barrier after in vitro ETEC infection, a change in claudin-4 expression was indeed detected, accompanied by a comparatively lower increase of cytokine expression after ETEC infection in the probiotic-fed group compared to the control group, a potentially significant finding regarding the mechanisms of diarrhea protection associated with E. faecium. The experiments of Lodenmann et al. were conceived to study the effects of ETEC infection in Ussing chambers. Nevertheless, it is possible that the effects of probiotics on TJ can protect the small intestine against further evolution of the intestinal disease.

The overall perception of the results of the article of Lodenmann et al. is that the microbiota is essential regulators of host physiology in the maintenance of a healthy intestine. Regarding ETEC pathogenesis, previous studies suggest a permeability increase (resistance decrease) of the intestinal barrier after ETEC infection, which is also associated with deleterious effects on the small intestinal mucosa. The present studies contribute to the understanding of ETEC colonization of the small intestine, particularly the early response of the host prior to all of the other events the produce diarrhea and disease after longer periods of infection as described in previous reports. Further studies would be necessary to clarify whether this early response of the small intestine in response to ETEC is actually generalizable to all Gram-negative bacteria and 
whether LPS is the trigger of this initial intestinal change. It is necessary to know more precisely the progress and outcome of ETEC (or Gram-negative bacterial) colonization and whether the initial changes described by Lodenmann et al. are or are not present as it would be important to develop tools to help improve human and animal health, as well as animal production.

\section{References}

1. Sears CL, Kaper JB. Enteric bacterial toxins: mechanisms of action and linkage to intestinal secretion. Microbiol Rev. 1996;5:167-215.

2. Lodemann U, Amasheh S, Radloff J, et al. Effects of ex vivo infection with ETEC on jejunal barrier properties and cytokine expression in probiotic-supplemented pigs. Dig Dis Sci. (Epub ahead of print). doi:10.1007/s10620-016-4413-x.

3. Awad WA, Aschenbach JR, Khayal B, Hess C, Hess M. Intestinal epithelial responses to Salmonella enterica serovar Enteritidis: effects on intestinal permeability and ion transport. Poult Sci. 2012;1:2949-2957.

4. Albin DM, Wubben JE, Rowlett JM, Tappenden KA, Nowak RA. Changes in small intestinal nutrient transport and barrier function after lipopolysaccharide exposure in two pig breeds. J Anim Sci. 2007;85:2517.

5. Bruins MJ, Cermak R, Kiers JL, van der Meulen J, van Amelsvoort JMM, van Klinken BJW. In vivo and in vitro effects of tea extracts on enterotoxigenic Escherichia coli-induced intestinal fluid loss in animal models. J Pediatr Gastroenterol Nutr. 2006;43:459-469.

6. Zhang W, Berberov EM, Freeling J, He D, Moxley RA, Francis DH. Significance of heat-stable and heat-labile enterotoxins in porcine colibacillosis in an additive model for pathogenicity studies. Infect Immun. 2006;1:3107-3114.

7. Hayden UL, Greenberg RN, Carey HV. Role of prostaglandins and enteric nerves in Escherichia coli heat-stable enterotoxin (STa)-induced intestinal secretion in pigs. Am $J$ Vet Res. 2017;57:211-215.

8. Capaldo CT, Farkas AE, Hilgarth RS, et al. Proinflammatory cytokine-induced tight junction remodeling through dynamic self-assembly of claudins. Mol Biol Cell. 2014;15:2710-2719.

9. Peterson LW, Artis D. Intestinal epithelial cells: regulators of barrier function and immune homeostasis. Nat Rev Immunol. 2014;25:141-153.

10. Gao Y, Han F, Huang X, Rong Y, Yi H, Wang Y. Changes in gut microbial populations, intestinal morphology, expression of tight junction proteins, and cytokine production between two pig breeds after challenge with Escherichia coli K88: a comparative study. J Anim Sci. 2013;1:5614-5625.

11. McLamb BL, Gibson AJ, Overman EL, Stahl C, Moeser AJ. Early weaning stress in pigs impairs innate mucosal immune responses to enterotoxigenic $E$. coli challenge and exacerbates intestinal injury and clinical disease. PLoS One. 2013;24:e59838.

12. Zeyner A, Boldt E. Effects of a probiotic Enterococcus faecium strain supplemented from birth to weaning on diarrhoea patterns and performance of piglets. J Anim Physiol Anim Nutr (Berl). 2006;90:25-31. 